\title{
Infection rate and spontaneous seroreversion of anti-hepatitis $C$ virus during the natural course of hepatitis $C$ virus infection in the general population
}

\author{
L A Kondili, P Chionne, A Costantino, U Villano, C Lo Noce, F Pannozzo, A Mele, \\ $S$ Giampaoli, M Rapicetta
}

Gut 2002;50:693-696

See end of article for authors' affiliations

\section{Correspondence to:} Dr M Rapicetta Department of Virology, Istituto Superiore di Sanità, $\checkmark$ le Regina Elena 299 00161 Rome, Italy; rapicett@iss.it

Accepted for publication 7 August 2001

\begin{abstract}
Background: Hepatitis $\mathrm{C}$ virus (HCV) infection is common worldwide but there are different prevalence rates in different countries. Data on the incidence of $\mathrm{HCV}$ in the general population are scarce. Spontaneous viral clearance occurs in 10-25\% of infected individuals after acute infection yet controversy exists regarding the frequency of spontaneous clearance during the natural course of HCV infection in the general population.

Aims: Anti-HCV prevalence, HCV infection rate, and the kinetics of anti-HCV were studied in a cross section of the general population of central Italy.

Study population and methods: Anti-HCV prevalence (EIA-3 Ortho, RIBA-3 Ortho Chiron) was estimated in 3884 randomly selected individuals. Infection rate and antibody kinetics were estimated in 2032 participants for whom a second blood sample was taken after a median follow up of seven years. HCV-RNA determination by polymerase chain reaction was performed on follow up sera.

Results: The overall confirmed anti-HCV prevalence was $2.4 \%$. Two participants seroconverted for anti-HCV, giving an overall infection rate of 1.4 cases per 10000 person years $195 \%$ confidence interval 0.2-5.2 per 10000 person years). Of the 36 individuals confirmed as anti-HCV positive at enrolment, seven (19.4\%) showed complete seroreversion. Seven $(87 \%)$ of the eight individuals with indeterminate results at enrolment were serologically non-reactive at the end of follow up. Of the 25 participants confirmed to be anti-HCV positive at both enrolment and follow up, $23(92.0 \%)$ with stable serological profiles tested positive for HCV-RNA at the end of follow up.

Conclusions: There is still a permanent risk, although low, of HCV spread in the general population in an area of low level endemicity. In this setting, a wide spectrum of modifications of viral and antibody patterns can be observed in HCV infected patients.
\end{abstract}

$\mathrm{H}$ epatitis C virus (HCV), first isolated in 1989, is a major cause of liver disease worldwide. In Europe, the prevalence of HCV antibodies has been reported to range from $1 \%$ to $1.4 \% .{ }^{1}$ With regard to incidence, most of the available data are from cross sectional studies of selected high risk groups. Specifically, the incidence per 100 person years among drug users was reported to be 6.4 in Baltimore, ${ }^{2} 6.2$ in Italy, ${ }^{3}$ and 11.7 in Switzerland. ${ }^{4}$ Data referring to the general population or to countries with different anti-HCV prevalence rates are scarce, although studies show that the annual number of newly acquired infections worldwide has decreased from approximately 180000 in the mid-1980s to 28000 in 1995, and the major factors contributing to this decrease have been accurate screening of blood products and the active modification of risk factors.

In many individuals, HCV infection leads to virus persistence and chronic liver disease, often resulting in liver cirrhosis and hepatocellular carcinoma. Persistent chronic infection has been observed in $75-80 \%$ of cases. ${ }^{6}$ Spontaneous clearance of the virus occurs in an estimated $10-25 \%$ of infected individuals after acute infection ${ }^{6}$ yet controversy exists regarding the existence and frequency of spontaneous antibody clearance during the natural course of chronic HCV infection (that is, seroreversion). Although this phenomenon has been reported in those undergoing haemodialysis, ${ }^{7}$ in immunosuppressed patients, ${ }^{8}$ and in immunocompetent individuals receiving interferon (IFN) therapy, ${ }^{9}$ its occurrence in the immunocompetent general population and the correlation with clearance of HCV viraemia have not been sufficiently documented.
The objective of the present study was to estimate anti-HCV prevalence, the risk of acquiring $\mathrm{HCV}$ infection, and the frequency and characteristics of anti-HCV seroreversion. To this end, we conducted a population based survey in an area of central Italy, retrospectively testing the sera of randomly selected individuals aged $20-69$ years.

\section{METHODS}

\section{Study population and sera}

The study population was that of an ongoing epidemiological survey of cardiovascular risk factors (MATISS project). ${ }^{10}$ Specifically, it consisted of residents of a geographical area located about $100 \mathrm{~km}$ southeast of Rome (central Italy) and comprised four adjacent towns (Sezze, Roccagorga, Bassiano, and Priverno). These subjects had been randomly selected from the electoral rolls (11 299 eligible persons) and represented a cross section of the general population aged 20-69 years. They were invited to participate in the survey by letter, and enrolment took place between June 1983 and March 1987. All participants were interviewed using a standard questionnaire containing information on age, sex, employment, level of education, and marital status.

The study was conducted using serum samples collected as part of the survey on cardiovascular risk factors. Anti-HCV

Abbreviations: $\mathrm{HCV}$, hepatitis $\mathrm{C}$ virus; anti-HCV, antibodies to hepatitis $C$ virus; IFN, interferon; $P C R$, polymerase chain reaction; $O R$, odds ratio. 


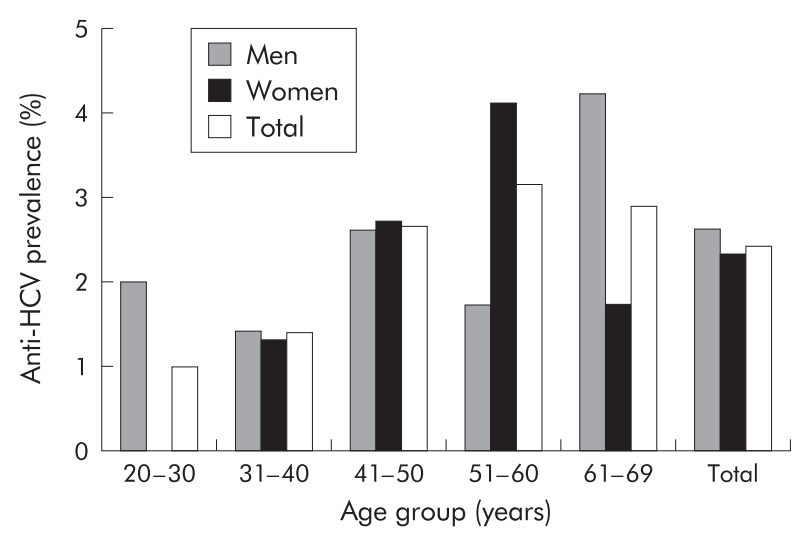

Figure 1 Prevalence of antibodies to hepatitis $\mathrm{C}$ virus (HCV) (RIBA-3 confirmed) by sex and age among 3884 subjects in an area of central Italy.

prevalence was estimated in serum samples (stored at $-20^{\circ} \mathrm{C}$ ) collected at enrolment (from 1983 to $1987 ; 3884$ subjects). HCV infection rate and the kinetics of anti-HCV were evaluated for the 2032 participants for whom a second serum sample (stored at $-70^{\circ}$ ) was obtained between 1993 and 1996 (52.3\% of the original 3884 participants). There were no significant differences in terms of age, sex, or employment when comparing participants at enrolment with those at follow up. Anti-HCV determination was performed on all serum samples whereas HCV-RNA determination was performed only on follow up sera. The study protocol conformed with the ethical guidelines of the Declaration of Helsinki (1975).

\section{HCV antibody and viral assays}

Testing for HCV antibodies was performed with a third generation enzyme immunoassay (EIA-3; Ortho HCV 3rd generation; Ortho Diagnostic Systems, Raritan, New Hampshire, USA) following the manufacturer's instructions. AntiHCV immunoreactivity was confirmed with a third generation immunoblot assay (RIBA-3; Chiron Corporation, Emeryville, California, USA, and Ortho Diagnostic Systems, Raritan, New Jersey, USA). RIBA determinations for anti-HCV positive sera were performed in the same run for the two serum samples (that is, samples obtained at enrolment and those obtained at follow up). HCV-RNA was determined for serum samples drawn in 1993-1996 and positive for anti-HCV using Cobas Amplicor 2.0 (Roche Diagnostic Systems, Branchburg, New Jersey, USA), following the manufacturer's instructions. Genotyping was performed by Innogenetics Line Probe Assay (Innogenetics, Zwijndrecht, Belgium) and by genotype specific polymerase chain reaction (PCR) in the core region using a modified version of the procedure proposed by Okamoto and colleagues, ${ }^{11}$ replacing the primer for subtype $2 \mathrm{~b}$ and including a new primer for subtype $2 \mathrm{c}$ and genotype 4 (reported to be circulating in Italy). ${ }^{12}$

\section{Statistical analysis}

Differences in proportions were evaluated using the $\chi^{2}$ test. A $\mathrm{p}$ value $<0.05$ was considered significant. Independent associations between HCV infection and individual sociodemographic characteristics were evaluated by multiple logistic regression analysis. ${ }^{13}$ In the multiple logistic model, anti-HCV positivity was the outcome variable and age, sex, level of education, employment, and marital status were independent variables. The reference category for odds ratio (OR) estimates was that of the most favourable level of exposure (that is, youngest age group, female, highest level of education, housewife, and never married) obtained by univariate analysis.

The incidence (infection rate) of HCV infection was calculated as the number of new infections per 10000 person years observed during follow up. Duration of follow up was calculated as the number of years between the date that the first blood sample was obtained and that of the second sample. The $95 \%$ confidence interval (CI) was calculated based on Poisson distribution.

\section{RESULTS}

At enrolment, 113 (2.9\%) of the 3884 participants were antiHCV positive when tested with EIA-3. When tested with RIBA-3, 93 of these 113 participants (82.3\%) tested positive: $18(15.9 \%)$ had indeterminate results and two tested negative ( $1.8 \%)$. Thus the overall confirmed anti-HCV prevalence was $2.4 \%(93 / 3884)$.

The age specific prevalence of anti-HCV antibodies by sex is shown in fig 1 . The overall anti-HCV prevalence was similar for males and females $(2.6 v 2.3 ; \mathrm{p}>0.05)$. Anti-HCV prevalence ranged from $0.98 \%$ among participants who were $20-30$ years of age to $3.14 \%$ among those aged 51-60 years, with a significant trend for an increase with age $\left(\chi^{2}\right.$ for linear trend $=6.4$, $\mathrm{p}=0.01)$.

When each variable was adjusted for the confounding effect of all other variables (age, sex, level of education, employment, marital status) by multiple logistic analysis, we observed that age greater than 45 years was the only variable independently associated with the presence of anti-HCV positivity (OR 2.65; 95\% CI 1.42-4.94).

\section{Infection rate of $\mathrm{HCV}$ infection}

A second serum sample was taken from 2032 of the 3884 participants (52.3\% response). During the median follow up of seven years (range 5.75-9.6), two of the participants seroconverted for anti-HCV antibodies (table 1), as confirmed by RIBA and by detection of HCV-RNA by PCR, representing an overall incidence of 1.4 cases per 10000 person years (95\% CI $0.2-5.2$ per 10000 person years). For both seroconverters, the HCV genotype was 2c. The age of the two seroconverters was 36 and 62 years, and both were apparently healthy during follow up. Neither had known risk factors for HCV infection during follow up, as determined by reviewing clinical records and speaking with their physicians.

Table 1 Changes (seroconversion and seroreversion) in anti-hepatitis C virus (HCV) reactivity among 2032 individuals for whom a second serum sample was available

\begin{tabular}{|c|c|c|c|c|}
\hline \multirow[b]{2}{*}{ First sample } & \multirow[b]{2}{*}{$\mathrm{n}$} & \multicolumn{3}{|l|}{ Second sample } \\
\hline & & $\begin{array}{l}\text { No of ElA reactive/RIBA positive } \\
(\text { No of PCR }+ \text { ) }\end{array}$ & $\begin{array}{l}\text { No of EIA reactive/RIBA } \\
\text { indeterminate (No of PCR+) }\end{array}$ & $\begin{array}{l}\text { No of EIA negative/RIBA negative } \\
(\text { No of PCR }+ \text { ) }\end{array}$ \\
\hline EIA reactive/RIBA positive & 36 & $25(23)^{*}$ & $4(2) \dagger$ & $7(0)$ \\
\hline EIA reactive/RIBA indeterminate & 8 & 1 (1) & 0 & 7 (0) \\
\hline EIA negative & 1988 & $2(2)$ & 0 & 1986 (ND) \\
\hline
\end{tabular}

*Stable anti-HCV serological profiles during follow up. †Decreased intensity of the bands during follow up.

‡Polymerase chain reaction (PCR) was not performed in the second sample if both the first and second samples tested EIA negative. 

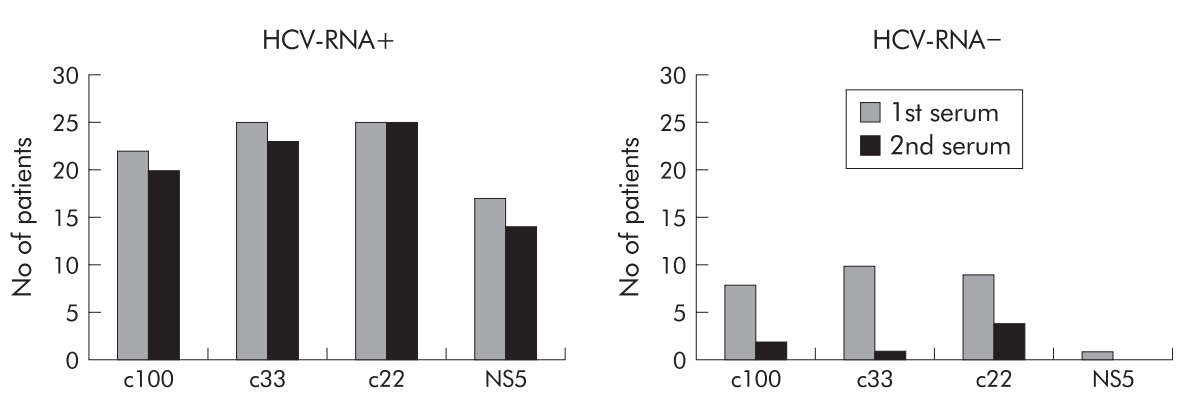

Figure 2 Modification of antibody pattern in follow up sera from 36 individuals positive to antibodies to hepatitis $C$ virus (RIBA-3 confirmed) according to the HCV-RNA positivity of the second serum sample.

\section{The kinetics of anti-HCV}

Table 1 and fig 2 illustrate the observed changes in anti-HCV reactivity during follow up and the correlation with the results of HCV-RNA determinations. Of the 36 individuals who were confirmed to be anti-HCV positive at enrolment, seven (19.4\%) showed complete seroreversion. Their median age was 56 years (range 45-66). All were healthy and asymptomatic for HCV infection. They had received neither antiviral nor immunosuppressive drugs during follow up (none of the anti-HCV positive cases had received these types of drugs). Of the eight serum samples with indeterminate results at enrolment, one (which was reactive only for the c22 band at enrolment) tested reactive for three bands (c11, c22, c33) at follow up. The remaining seven samples (six of which had been reactive only for the c22 band and one only for the c 33 band) were not reactive for any band at follow up. In four of the 36 participants with RIBA-3 pattern reactive to 3-4 bands at enrolment, we observed indeterminate results at follow up, with positivity for only the c22 band. Two of these four sera tested positive for HCV-RNA.

All 14 participants with negative results by EIA-3 and RIBA-3 at follow up were negative for HCV-RNA. Of the 25 participants confirmed to be anti-HCV positive at both enrolment and follow up, 23 (92.0\%) tested positive for HCV-RNA: 14 were assigned to genotype $2 \mathrm{c}$ and the others to genotype $\mathrm{lb}$. The sera of all of these participants had nearly stable serological profiles from enrolment to the end of follow up whereas two of the 25 participants (13\%) who tested HCV-RNA negative showed a decreased number and intensity of bands.

Figure 2 illustrates the results relative to the single antibody reactivities by RIBA-3 in HCV-RNA positive and HCV-RNA negative participants who were confirmed to be anti-HCV positive at enrolment. The level of positivity decreased in follow up sera for all antigens in both groups. This decrease was less marked for the c22 band than for the other bands, indicating a greater stability for the antibodies against this structural protein.

\section{DISCUSSION}

HCV seroprevalence studies conducted among randomly selected community based samples of the general population from different areas of Italy have shown different anti-HCV prevalence rates. Low rates (range $0.8-1.51 \%$ ) were observed when the first generation anti-HCV screening assay was used $^{101415}$ whereas the rates observed with the more sensitive third generation ELISA ranged from $2.7 \%$ to $26 \%$. $^{16-19}$ The $2.4 \%$ prevalence found in our study indicates that the endemicity level of HCV infection is low in the area studied. In this epidemiological setting, the incidence was 1.4/10 000 person years. As the study population was that of a survey originally designed to investigate the prevalence of cardiovascular diseases, no systematic effort was made to record risk factors for parentally transmitted infections. However, the fact that the study population was not selected for testing on the basis of the presumed risk of infection, in addition to the procedure adopted for random sampling, support the absence of selection bias.

Following the introduction of systematic screening of blood donations for anti-HCV, many studies focused on parenteral routes of transmission and community acquired infections. As surrogate indicators of the spread of HCV infection in the community, we evaluated the effect of sociodemographic characteristics and educational level on anti-HCV prevalence. No association was found for any of these factors with the exception of age greater than 45 years. The observed age trend for anti-HCV is similar to trends reported by other surveys performed in areas that are hyperendemic for $\mathrm{HCV}^{19}$ confirming that the cohort effect (that is, decreased risk of infection with decreasing age) is independent of the level of endemicity.

Although the incidence of HCV has been previously evaluated among selected population groups (for example, blood donors and high risk groups such as parenteral drug users, haemodialysis patients, and healthcare workers), ${ }^{2-4} 72021$ to the best of our knowledge only one other population based incidence study has been conducted to date. However, this study was carried out in a hyperendemic area $(26 \%$ prevalence). ${ }^{22}$ The present study was conducted in a randomly selected community based sample of the general population in an area with a low level of endemicity in which the anti-HCV prevalence rates are similar to those reported in most areas of Italy and Eastern Europe. ${ }^{23}{ }^{24} \mathrm{HCV}$ incidence was found to be similar to that reported for healthy blood donors ( $1.4 v 1$ per 10000 person years $)^{20}$ and lower than that observed in hyperendemic areas in Italy (3.42 per 10000 person years). ${ }^{22}$ In our study, among individuals for whom no second sample was available, the prevalence of anti-HCV was higher than that among those for whom the infection rate was computed. There were no significant differences in terms of age, sex, or employment when these two groups of participants were compared (see methods section). A decrease in HCV infection was observed in the general population, probably as a result of the use of disposable needles and a decrease in injection drug use. For these reasons, we assume that in our study population of healthy individuals, in the absence of major risk factors (drug users), the HCV infection rate was probably the same, independent of the different prevalence values that were the consequence of exposure before 1983-1987. Our finding of only two seroconversions during follow up clearly indicates that the spread of HCV infection among adults in this area is relatively low. However, HCV infection must not be underestimated because there is still a permanent risk of transmission among members of the healthy general population. ${ }^{25}$

We also estimated the frequency of spontaneous anti-HCV seroreversion in a homogeneous population of immunocompetent subjects not subjected to treatment with IFN- $\alpha$ or immunosuppressors. In an infected host, the virus can cause short term acute infection or can establish long term persistence. In most individuals, viral RNA and antibodies persist for very long periods, perhaps for a lifetime, as shown by previous data on non-treated patients with chronic HCV infection. ${ }^{6}$ However, it has been hypothesised that HCV infection is not always characterised by the persistence of the virus and antibodies. ${ }^{9}$ Antibody seroreversion rates of $2-10 \%$ were found in a cohort of individuals with post-transfusion $\mathrm{HCV}$ 
infection, ${ }^{26}$ in those from a hyperendemic area, ${ }^{22}$ and in haemodialysis patients. ${ }^{7}$ To date, there are no data on antibody seroreversion rates among the general population from a low level endemic area.

In our study, $19.4 \%$ of participants with RIBA confirmed results at enrolment (reactivity to 2-4 bands) and $87 \%$ of those with indeterminate results (reactivity to one band) were serologically non-reactive to both the immunoenzymatic and immunoblot test after a median follow up of seven years. Furthermore, a decreased level of positivity was observed in four of the 36 RIBA confirmed participants at enrolment, two of whom were still HCV-RNA positive. These data are consistent with those reported for a high risk population. ${ }^{7}$ In this population (217 haemodialysis patients), which was followed for 12 years, the yearly anti-HCV prevalence rates tended to decrease over time, from $43.6 \%$ to $29.2 \%$ over the entire study period.

Other recent studies have found an almost complete correlation between complete antibody seroreversion and lack of HCV viraemia among individuals exposed to HCV. ${ }^{9}$ Full or partial seroreversion in these individuals was related to loss of antigenic stimulation or associated with the presence of a low viraemia level (that is, below the threshold necessary for maintaining an efficient humoral immune response). In contrast, a stable serological profile was found to be associated with continuous antigenic stimulation due to chronic replication of the virus. ${ }^{27}$

Seroreversion occurred in individuals with a median age of 56 years (range 45-66) in a low endemic locality, and in a population with a low probability of exposure to the highest risk factors and with a low infection rate (only two seroconversions). For these reasons, there is little likelihood that in these cases seroreversion represented recovery from a recent acute infection. Moreover, the presence in our cohort of subjects with partial seroreversion in the absence of viraemia (suggesting recovery from past infection) after a median period of seven years of follow up indicates that individuals who clear HCV may have a gradual loss of antibodies. As also observed in a recent study, after a period of time following transfusion associated hepatitis (non-A, non B, and C), some patients showed no serological or molecular evidence of their earlier HCV infection. ${ }^{26}$ The difference in terms of the frequency of seroreversion $(19.1 \% \vee 7 \%)$ between the above study and ours could be due to the different transmission routes of infection or simply to chance.

Our data also suggest, in accordance with other studies, that the $\mathrm{c} 22$ band, with respect to the other bands, more frequently represents the last marker to become negative; however, the presence of only the c22 band does not necessarily indicate seroreversion.

In conclusion, there is still a permanent risk of HCV spread in the general population of a low level endemic area. A wide spectrum of modifications of viral and antibody serological patterns can be observed during the natural course of HCV infection. Both host related and viral factors, in addition to environmental factors such as level of exposure to infection, may be responsible for these different patterns.

\section{ACKNOWLEDGEMENTS}

The research was conducted within the Project CUORE "Epidemiology and prevention of ischemic heart disease" partly supported by funds from the Ministry of Health. The study is named MATISS "Malattie cardiovascolari Aterosclerotiche Istituto Superiore di Sanità". Members of the MATISS study are: S Giampaoli, F Pannozzo, MF Vescio, P Zuccaro, P Caiola De Sanctis, F Dima, C Lo Noce, A Menditto, A Minoprio, and A Santaquilani. The authors thank Mark Kanieff for editorial assistance and text revision, Romina Tomasetto for editorial assistance, and Luigia Mauro for secretarial assistance. The study was supported by the Istituto Superiore di Sanità "National Viral Hepatitis" Project.

\section{Authors' affiliations}

LA Kondili, P Chionne, A Costantino, U Villano, M Rapicetta, Department of Virology, Istituto Superiore di Sanità, Rome, Italy

C Lo Noce, A Mele, S Gianpaoli, Department of Epidemiology, Istituto Superiore di Sanità, Rome, Italy

F Pannozzo, USL, Latina, Italy

\section{REFERENCES}

1 Sarbah SA, Younossi ZM. Hepatitis C: an update on the silent epidemic J Clin Gastroenterol 2000;30:125-43.

2 Villano SA, Vlahov D, Nelson KE, et al. Incidence and risk factors for hepatitis $\mathrm{C}$ among injection drug users in Baltimore, Maryland. J Clin Microbiol 1997:35:3274-7.

3 Galeazzi B, Tufano A, Barbierato E, et al. Hepatitis C virus infection in Italian intravenous drug users: epidemiological and clinical aspects. Liver 1995; 15:209-12.

4 Chamot E, de Saussure P, Hirschel B, et al. Incidence of hepatitis C, hepatitis $B$ and HIV infections among drug users in a methadone-maintenance programme. AIDS 1992;6:429-31.

5 Alter MJ, Kruszon-Moran D, Nainan OV, et al. The prevalence of hepatitis C virus infection in the United States, 1988 through 1994. N Engl J Med 1999:341:556-62.

6 Alter MJ, Margolis HS, Krawczynski K, et al. The natural history of community-acquired hepatitis $C$ in the United States. The Sentinel Counties Chronic non-A, non-B Hepatitis Study Team. N Engl J Med 1992;327: 1899-905

7 Simon N, Courouce AM, Lemarrec $N$, et al. A twelve year natural history of hepatitis $C$ virus infection in hemodialyzed patients. Kidney Int 1994:46:504-11

8 Goffin E, Pirson Y, Cornu C, et al. Outcome of HCV infection after renal transplantation. Kidney Int 1994:45:551-5.

9 Lefrère $\mathrm{JJ}$, Guiramand $\mathrm{S}$, Lefrère $\mathrm{F}$, et al. Full or partial seroreversion in patients infected by hepatitis C virus. J Infect Dis 1997;175:316-22.

10 Gianpaoli S, Poce A, Sciarra F, et al. Change in cardiovascular risk factors during a 10 year community intervention program. Acta Cardiol 1997:5:411-22.

11 Okamoto $\mathbf{H}$, Sugiyama H, Okada S, et al. Typing hepatitis $\mathrm{C}$ virus by polymerase chain reaction with type-specific primers: application to clinical surveys and tracing infectious sources. J Gen Viro 1992;73:673-9

12 Spada E, Ciccaglione AR, Dettori S, et al. Genotyping HCV isolates from Italy by type-specific PCR assay in the core region. Res Virol 1998;149:209-18.

13 Kleinbaum DG, Kupper LL, Chambless LE. Logistic regression analysis of epidemiological data. Theory and practice. Commun Stat Theor M 1982;71:485-547.

14 Rapicetta $M$, Attili AF, Mele A, et al. Prevalence of hepatitis $C$ virus antibodies and hepatitis $C$ virus-RNA in urban population. J Med Virol 1992;37:87-92

15 Chiaramonte M, Stroffolini T, Caporaso N, et al. Hepatitis-C virus infection in ltaly: a multicentric sero-epidemiological study la report from the HCV study group of the Italian Association for the Study of the Liver). Ital J Gastroenterol 1991;23:555-8.

16 Coppola RC, Masia G, Pradat P, et al. Impact of hepatitis $C$ virus infection on healthy subjects on an Italian island. J Viral Hepat 2000;7:130-7.

17 Maggi G, Armitano S, Brambilla L, et al. Hepatitis $C$ infection in an Italian population not selected for risk factors. Liver 1999;19:427-31.

18 Maio G, d'Argenio P, Stroffolini T, et al. Hepatitis $C$ virus infection and alanine transaminase levels in the general population: a survey in a southern Italian town. J Hepatol 2000;33:1 16-20.

19 Guadagnino V, Stroffolini T, Rapicetta M, et al. Prevalence, risk factors, and genotype distribution of hepatitis $C$ virus infection in the general population: a community-based survey in southern Italy. Hepatology 1997;26:1006-11

20 Prati D, Capelli C, Silvani C, et al. The incidence and risk factors of community-acquired hepatitis $\mathrm{C}$ in a cohort of Italian blood donors. Hepatology 1997;25:702-4

21 Stroffolini T, Marzolini A, Palumbo F, et al. Incidence of non-A, non-B and HCV positive hepatitis in healthcare workers in Italy. J Hosp Infect 1996; 33:131-7.

22 Osella AR, Misciagna G, Leone A, et al. Epidemiology of hepatitis C virus infection in an area of Southern Italy. J Hepatol 1997;27:30-5.

23 Bellentani S, Pozzato G, Saccoccio G, et al. Clinical course and risk factors of hepatitis $C$ virus related liver disease in the general population: report from the Dionysos study. Gut 1999:44:874-80.

24 Trepo C, Pradat P. Hepatitis C virus infection in Western Europe. J Hepatol 1999;31:80-3.

25 Mele A, Tosti ME, Marzolini A, et al. Prevention of hepatitis $C$ in Italy: lessons from surveillance of type-specific acute viral hepatitis. J Viral Hepat 2000;7:30-5.

26 Seeff LB, Hollinger FB, Alter HJ, et al. Long-term mortality and morbidity of transfusion-associated non-A non-B, and type $C$ hepatitis: a National heart, Lung, and Blood Institute collaborative study. Hepatology $2001 ; 33: 455-63$

27 Lanotte P, Dubois F, Le Pogam S, et al. The kinetics of antibodies against hepatitis $C$ virus may predict viral clearance in exposed hemophiliacs. J Infect Dis 1998 178:556-9. 